\title{
Análisis de contenido y teorias subyacentes en los textos españoles de referencia sobre Didáctica General
}

\section{Analysis of content and underlying theories in Spanish reference texts on General Didactics}

\begin{abstract}
Dra. Cristina MORAL SANTAELLA. Profesora Titular. Universidad de Granada (cmora@ugr.es).
Dr. Agustín DE LA HERRÁN GASCÓN. Profesor Titular. Universidad Autónoma de Madrid (agustin.delaherran@uam.es).
\end{abstract}

\section{Resumen:}

Los resultados de los Informes Pisa 2019 confirman que el sistema educativo español sigue estancado al finalizar la enseñanza obligatoria. Esta situación requiere una autocrítica de todos los agentes implicados. Uno de los colectivos concernidos son los profesores universitarios que preparan a los que serán futuros docentes. Entre ellos, se encuentran los que enseñan Didáctica General, que facilitan una formación didáctica básica y polivalente a los futuros docentes. Para ello, suelen tener en cuenta manuales clásicos y recientes y otros textos de referencia sobre teoría y práctica de la enseñanza, que contribuyen a definir la disciplina. El objetivo del estudio es conocer si estas obras preparan a los futuros profesores en com- petencias docentes y verificar su potencial didáctico y profesionalizador de la teoría de la enseñanza que transmiten. Para dar respuesta a este objetivo, se optó por un estudio documental de carácter descriptivo, de 35 obras de referencia de Didáctica General, entre las que se incluyeron tanto manuales como textos utilizados como fuentes de conocimiento didáctico. Sobre esos textos se ha realizado un análisis de contenido de su estructura y composición. Los resultados muestran una tendencia a valorar los aspectos teóricos sobre los competenciales y a ordenar sus capítulos desde una tradición técnico-administrativa, en vez de hacerlo desde una aproximación conceptual y semántica enfocada al diseño de una experiencia de aprendizaje significativa. Como síntesis apli-

Fecha de recepción de la versión definitiva de este artículo: 10-05-2021.

Cómo citar este artículo: Moral Santaella, C. y De la Herrán Gascón, A. (2021). Análisis de contenido y teorías subyacentes en los textos españoles de referencia sobre Didáctica General | Analysis of content and underlying theories in Spanish reference texts on General Didactics. Revista Española de Pedagogía, 79 (280), 437-455. https://doi. org/10.22550/REP79-3-2021-01

https://revistadepedagogia.org/ 
cada, se aportan recomendaciones para elaborar manuales de Didáctica General, orientados a la formación inicial del profesorado, desde un enfoque más competencial y menos academicista.

Descriptores: Didáctica General, obra de referencia, formación de profesores, competencia, profesión docente.

\section{Abstract:}

The results of the 2019 Pisa Reports confirm that the Spanish education system continues to stagnate after compulsory education has been completed. This situation calls for self-criticism on the part of all actors involved. One of the groups concerned are the university professors and lecturers who provide training for prospective teachers. Among them are those who teach General Didactics. They provide basic and multi-purpose training on teaching methods for prospective teachers. For this purpose, they usually consider traditional and recent textbooks and other reference texts on teaching theory and practice, which help to define the discipline. The aim of the study is to find out whether these works train and equip prospective teachers with teaching competences and to verify their educational and professional potential with regard to the teaching theory they convey. In order to fulfil this aim, a descriptive documentary study of 35 reference works on General Didactics was carried out, including both textbooks and texts used as sources of didactic knowledge. The content of the structure and composition of these texts was analysed. The results show a tendency to prioritise theoretical aspects over competences and to order chapters based on a technical and administrative tradition, rather than a conceptual and semantic approach focused on designing a meaningful learning experience. As an applied summary, recommendations are provided for the development of textbooks on General Didactics, aimed at initial teacher training, from a more competence-based and less academic approach.

Keywords: General Didactics, reference work, teacher training, competence, teaching profession.

\section{Introducción}

La autocrítica es necesaria en educación e investigación. Puede fundamentar rectificaciones y mejorar procesos y resultados. Cuando se trata de quienes forman a los futuros profesores, esta acción es un imperativo, aunque se realice poco. En este campo formativo se sitúa este trabajo.

Si la formación de docentes y la educación de calidad están relacionadas (Con-

sejo Escolar del Estado, 2015), también podrían estarlo los malos resultados de los informes PISA de 2020, la estancada mediocridad del sistema educativo español y quienes preparan a los futuros docentes. El profesorado universitario que forma a los futuros profesores, como observador y agente concernido, debería reflexionar. En un texto de autocrítica pedagógica, Bolívar y Pérez (2019) abordaron las «políticas educativas sobre el profesorado», consi- 
derando los créditos, las regulaciones, las reformas legislativas, los trámites, la selección, las competencias profesionales, la ética, etc. En un campo tan complejo como la educación y la enseñanza, cabe analizar, complementariamente, el modelo de formación inicial del profesorado vigente en España.

Los programas se estructuran en asignaturas teóricas, donde se adquiere el conocimiento básico de la profesión, y en uno 0 más periodos de prácticas, donde se observará y contrastará parte de ese conocimiento. Se supone que, así —en un año, los docentes de secundaria-, adquirirán las competencias necesarias para llevar a cabo una buena práctica docente. Estudios recientes cuestionan este modelo, apuntando a un necesario cambio formativo. La objeción es la escasa conexión entre la teoría trasmitida en la facultad y la práctica de la enseñanza. Se cuestiona el potencial profesionalizador en competencias docentes de estos programas, ya que muchos futuros profesores consideran que las asignaturas ordinarias no se las ofrecen, pese a lo prometido en sus guías docentes. Además, consideran que el aprendizaje de las competencias docentes ocurre durante el prácticum, aunque se detecte un hiato entre el prácticum y las demás asignaturas, y una debilidad de los instrumentos formativos que los conectan. Esto hace que su coherencia didáctica sea escasa y mejorable, si de desarrollar competencias se trata (Egido y López, 2016; Gairín et al., 2019).

Se podrían identificar algunas variables como posibles causas explicativas de este problema. Entre todas, destaca una variable radical o causal: el tipo de conocimiento desde el que los formadores preparan a los futuros profesores. Shulman (2005) apuntaban a que uno de los conocimientos básicos de los docentes es el llamado «general pedagogical knowledge», traducido por Bolívar como «conocimiento didáctico general». Este ámbito encajaría con el corpus de la Didáctica General o Polivalente. Se refiere a la teoría y la práctica de la enseñanza para la formación, aportando, entre otras, las competencias para el diseño y desarrollo de experiencias de aprendizaje significativas y educativas.

Las obras de referencia de Didáctica General recogen y sintetizan este conocimiento para la formación del profesorado. Ahora bien, iproporcionan un carácter profesionalizador encaminado a la formación en competencias y prácticas docentes básicas/centrales que se demandan en la actualidad, o se plantean desde una aproximación academicista desconectada de la práctica? Y otra cuestión esencial: ¿desde qué teoría de la enseñanza se construyen? Las respuestas a estas preguntas fundamentan estas inquisiciones, complementarias al trabajo de Heredia (2015), si bien este investigador estudia la organización interna de manuales asimilables al ámbito de la Didáctica General con una perspectiva histórica y epistemológica y, en nuestro caso, se analizan cuestionando tanto la teoría de la enseñanza que les sirve de modelo como la orientación académica o profesionalizadora que les da fundamento. 


\section{Fundamentos para una teoría de la enseñanza}

El docente, como otros profesionales, necesita un conocimiento básico y aplicable. La Didáctica General debería aportar, por un lado, los saberes y competencias esenciales para desarrollar una enseñanza educadora. Y, por otro, permitirle construir, desde su experiencia, una razón profesional y un acervo propio y fundamentado (episteme) para el diseño y desarrollo curricular.

El diseño curricular, centrado en la programación de experiencias didácticas, requiere una reflexión detenida sobre los componentes curriculares y su relación con el aprendizaje y la formación. Su reflexión no es sencilla, al relacionar, con un sentido educativo y pertinente, al menos, los elementos definidos por Tyler (1949) -objetivos, actividad y control-evaluación-, los «common places» de Schwab (1970) — profesor, alumnos, contenido y matriz social- y las competencias claves, como vertebradoras de la educación (Council of the European Union, 2018). Desde aquí, caben diseños innovadores, combinando o priorizando unos elementos sobre otros, para conseguir un aprendizaje significativo, profundo y relevante en los alumnos (Darling-Hammond y Oakes, 2019).

Para poder programar, es necesario comprender el significado del «aprendizaje», tanto como concepto asociado a una reflexión previa sobre la naturaleza y forma del conocimiento humano, como sobre su creación y organización cognoscitiva. La neurociencia y la psicología cognitiva aportan bases previas para la formación desde la Didáctica General, sobre el conocimiento humano originado desde un sistema de procesamiento de información no mecánico ni objetivo, sino subjetivo y reconstructivo y sustentado en la experiencia de cada sujeto (Sousa, 2017; Weinstein y Sumeracki, 2019). El conocimiento se origina en la misma experiencia de aprendizaje, en un proceso de transformación de la experiencia mediante un ciclo de acción/reflexión-experiencia/abstracción y organizándose en la mente mediante esquemas y estructuras conceptuales que establecen relaciones conceptuales significativas. Este conocimiento conceptual permite retener, recordar y transferir lo aprendido a la resolución de problemas. La retención y el recuerdo-identificación de la información es considerada un paso esencial para la trasferencia en el aprendizaje; lo malo es cuando se convierten en los fines últimos de la enseñanza, y no en un medio para la trasferencia de lo aprendido a situaciones $\mathrm{y}$ contextos funcionales.

La búsqueda de un aprendizaje significativo y aplicable, frente a un aprendizaje retentivo, no ha primado en la historia de la enseñanza. Sigue vigente, en muchos contextos, la observación freireana, que concebía el aprendizaje escolar como un proceso rutinario de almacenamiento de información, en el que primaba la domesticación y la memorización sin significado. Todavía se prioriza un aprendizaje representacional, o de adquisición de etiquetas, para replicar lo transmitido por el profesor, en vez de una adquisición de conceptos generadores de estructuras y esquemas 
conceptuales para la transferencia, que permitan al alumno razonar y desenvolverse con independencia.

Este tradicionalismo motivó el surgimiento de los planteamientos experimentalistas promovidos por Dewey (1902), que consideraron la experimentación, la actividad y el descubrimiento como bases del aprendizaje formativo. De este enfoque surgieron metodologías en las que predominaba la manipulación y la actividad como vías para descubrir y aprender conceptos. Pero se ha constatado que la mera experiencia del alumno no garantiza el descubrimiento de conceptos y el aprendizaje significativo. Se precisan circunstancias adecuadas y una enseñanza congruente. Para Novak (2010), en la teoría de la enseñanza vía acción y experimentación se ha ignorado el proceso de aprendizaje y la estructura en que se asienta. Para Walter y Soltis (2004), esta es la raíz del enfrentamiento entre los teóricos del currículo progresistas y tradicionales.

Los progresistas se oponen a las prácticas de memorización no significativa, y generan una gran variedad de propuestas. Por ejemplo, currículos alternativos, construidos alrededor del interés y necesidades del alumno, la actividad, la experiencia, la realización de tareas, el trabajo manual, etc. Algunos esperan que los alumnos generen su propio currículo, desde sus propios intereses (Walter y Soltis, 2004). En este enfrentamiento, el contenido a enseñar se ha desprestigiado hasta desaparecer como elemento esencial en los diseños didácticos.
En la didáctica española de los años 60, el contenido tuvo un cierto protagonismo: se consideraba el elemento clave del proceso de aprendizaje, porque la estructura conceptual era importante. Esta orientación estuvo amenazada por el movimiento en el que, al priorizarse la tarea 0 actividad, se relegaba el contenido. De hecho, llegó a desgajarse de la base de conocimiento que definía al profesional de la docencia, desapareciendo de los programas de formación y de las obras de referencia de Didáctica General, diluyéndose en los objetivos y la evaluación. Incluso, cuando se preguntaba «ipor qué enseñar?», se respondía considerando los objetivos educativos, no los contenidos (Rodríguez-Diéguez, 1980).

Hoy, lo importante son las competencias, la actividad, la experiencia en el aprendizaje. Las competencias propuestas por la European Commission (2004) irrumpieron con fuerza en el contexto español (Bolívar, 2010) y se asimilaron con celeridad. En el modelo competencial de enseñanza, las materias contribuyen a su desarrollo, se ponen en función suya, y la relevancia, adquisición y uso de saberes se asimila a lo tradicional.

Ante esta discusión dual, cabría preguntarse ¿cuál es el propósito de la educación? Ampliamente, hacer ciudadanos felices, acabar con la injusticia, con la desigualdad, contribuir al desarrollo social, etc. Más restrictivamente, el desarrollo de la persona mediante el aprendizaje (Walter y Soltis, 2004), para que cada alumno pueda pensar, sentir y actuar autónoma y creativamente 
(Novak, 2010). Se trata, pues, de superar un aprendizaje memorístico, sin caer en las incoherencias de un aprendizaje basado en la competencia y la experiencia. Para su programación, los componentes de la enseñanza (alumnos, profesor, contenido, clima, objetivos, actividad, evaluación y competencia) deben ser combinados para conseguir que el alumno se eduque en el pensar, sentir y actuar (Novak, 2010), apoyándose en los descubrimientos de la Neurociencia y la Psicología Cognitiva (Sousa, 2017; Weinstein y Sumeracki, 2019).

El orden y la organización de los componentes de la enseñanza favorecerán 0 no la orientación didáctica al aprendizaje. Una forma podría ser la siguiente: primero, todo profesor debe partir considerando al aprendiz y la necesidad de fomentar un aprendizaje personalizado y una educación que conecte y armonice sus emociones, intereses, motivaciones, estilos, contextos, experiencias, etc. A continuación, aquello de lo que trata la enseñanza 0 contenido didáctico (saberes disciplinares), reflexionando sobre su selección y organización, las preguntas centrales que lo hacen accesible y sus posibilidades de transferencia y uso (McTighe y Willis, 2019). En tercer lugar, cabría preguntarse por el sentido de sus aprendizajes, especificando los objetivos de la actuación, en función del contenido seleccionado y la estructura conceptual identificada. Para ello, se pueden utilizar taxonomías solventes de objetivos de aprendizaje, como la de Anderson y Krathwohl (2001), recientemente valorada desde la Neurociencia por Sousa (2017).
En ella, se progresa desde objetivos de recuerdo a objetivos de evaluación, diseño y creación. En cuarto lugar, el proceso de control del diseño de objetivos debe ser especificado para ir evaluando la progresión en el aprendizaje, facilitando el feedback necesario y la evaluación formativa precisa para un aprendizaje personalizado (Hattie y Clarke, 2019). En quinto lugar, una vez construido el esqueleto que da forma al diseño, se podrá avanzar identificando el sistema de relaciones creado en clase y la metodología didáctica para la acción y recreación del pensamiento, sentimiento y actuación de los alumnos (Ritchart y Church, 2020), que se verán enriquecidos por la diversidad de competencias clave desarrolladas desde las propuestas metodológicas, tareas y actividades de clase (Bolívar, 2010).

La estructura anterior es flexible, variable y susceptible de innovación. No obstante, incluir el contenido como base del proceso de programación de la enseñanza optimiza la articulación entre la Didáctica General y Específicas. Supone trascender el exacerbado «pedagogismo» de los años 1980, más interesado en valorar cómo se enseñaba que lo que se enseñaba, a quién y cuándo se enseñaba, y ampliamente rechazado por el profesorado de Secundaria (Bolívar, 2005). Implica dar sentido, desde una enseñanza activa y congruente, a la experiencia de aprendizaje formativo del alumno, poniendo aquello de lo que la enseñanza y el aprendizaje tratan como centro de atención formativa.

En síntesis: las recomendaciones e imperativos actuales sobre formación del 
profesorado destacan un modelo de enseñanza basado en competencias. El conocimiento que se transmite a los futuros profesores desde la Didáctica General debería encajar con ellas. En caso contrario, el conocimiento transmitido como base de su actuación será inútil y con pocas posibilidades de convertirse en práctico y profesionalizador. Ante esta duda, el presente estudio tiene como objetivo general conocer si las obras de referencia para la Didáctica General preparan a los futuros docentes en competencias didácticas y verificar hasta qué punto pueden considerarse, en este sentido, con potencial profesionalizador. Los objetivos específicos son tres: (1) verificar la teoría de la enseñanza que les da fundamento, comprobando el contenido que abordan; (2) conocer la estructura que ordena el contenido de los textos estudiados, y (3) colegir si el contenido que se transmite en estas obras mantiene un orden interno y coordinado.

\section{Metodología}

Para responder a los objetivos, el estudio se apoya en una investigación documental, que explora el fundamento de los textos de referencia de Didáctica General, respondiendo a las preguntas características de la investigación descriptiva: «qué, cómo y quién del fenómeno estudiado» (Hall, 2020, p. 35). Se revisaron 35 textos, seleccionados siguiendo tres criterios: haberse publicado desde los años 1980, cuando la Didáctica General comienza a considerarse un conocimiento científico asociado a estudios curriculares (Bolívar, 2008); haber sido
0 seguir siendo referentes en la elaboración de Guías Docentes de la asignatura de Didáctica General de numerosas universidades españolas, o abarcar el contenido específico de la Didáctica General.

Se describirán desde las siguientes categorías de análisis:

- Tipo de autoría: individual o coordinada.

- Temáticas abordadas:

a) Epistemología Didáctica/currículo: Didáctica como disciplina científica, conceptualización de Didáctica/currículo, teorías curriculares, tipos, modelos, paradigmas curriculares, teoría-práctica del currículum y reforma curricular, currículum y escuela, aproximaciones de investigación en didáctica.

b) Diseño del currículo: tipos de diseño didáctico, componentes curriculares, concreciones curriculares.

c) Profesorado: capital profesional, funciones, responsabilidades, autenticidad, identidad, autonomía.

d) Alumnado: personalización, motivación, estilos de aprendizaje, inteligencias, autonomía, identidad, metacognición, contexto.

e) Contenidos: selección, organización, secuenciación, aproximaciones disciplinares y globales. 
f) Objetivos: tipos y niveles de generalidad, taxonomías de objetivos de aprendizaje, diseño de objetivos.

g) Competencias: tipos de competencias, función de competencias, organización del diseño didáctico en torno a competencias.

h) Mezcla de elementos curriculares -capítulo donde se combinan los componentes curriculares, objetivos, contenidos, competencias, metodología, recursos y evaluación.

i) Metodología didáctica: cómo enseñar, tipos de metodologías (directa, colaborativa, indagadora, etc.), modelos de enseñanza-aprendizaje.

j) Recursos didácticos: tipos de recursos con y sin TIC, utilidad y aplicación de medios de enseñanza, didáctica y medios.

k) Evaluación didáctica: tipos de evaluación, procedimientos, instrumentos, evaluación de la enseñanza, evaluación del aprendizaje, evaluación del contexto de enseñanzaaprendizaje, implicaciones de la evaluación.

l) Técnicas: técnicas y estrategias facilitadoras del aprendizaje.

m) Clima de aula: gestión del clima del aula, interacción, comunicación, autoridad del profesor, resolución de conflictos, sistema de relaciones, gestión y organización del contexto de aprendizaje.

n) Desarrollo docente e innovación didáctica: procesos de mejora y cambio escolar, desarrollo profesional docente.

ñ) Ejemplos de diseño: casos para estudio y ejemplos de diseños didácticos.

- Número de capítulos relativos a cada temática.

- Orden de los capítulos.

El examen no entra en la profundidad de los contenidos de cada obra, pues se considera suficiente para responder a los objetivos. Algunos no fueron concebidos como manuales, pero han sido textos de referencia generalmente utilizados como fuentes de conocimiento didáctico básico y aplicado. Es el caso de Gimeno (1981), Gimeno y Pérez Gómez (1992), Escudero (1999) o Bolívar (2008), aunque unos son más amplios y otros más específicos, han dado fundamento al conocimiento didáctico. De aquí que puedan considerarse obras de referencia para la enseñanza de la Didáctica General de los futuros docentes. Con independencia de su mayor o menor uso, han determinado a la Didáctica y continúan marcando tendencia, a nivel nacional. Se analizan un total de 35 textos y 435 capítulos.

Los datos bibliográficos de los textos estudiados no se encuentran en el apartado "Referencias bibliográficas», ya que se consideran datos del estudio. Pueden encontrarse en la Tabla 1: 
Análisis de contenido y teorías subyacentes en los textos españoles de referencia sobre...

TABLA 1. Textos de referencia de Didáctica General revisados.

\begin{tabular}{|c|c|c|c|c|}
\hline N.o & Año & $\begin{array}{c}\text { Autores/ } \\
\text { Coordinadores }\end{array}$ & Título & $\begin{array}{l}\text { Lugar publica- } \\
\text { ción / Editorial }\end{array}$ \\
\hline 1 & 1980 & Rodríguez-Diéguez, J. L. & Didáctica General & Madrid: Cincel \\
\hline 2 & 1981 & Gimeno, J. & $\begin{array}{l}\text { Teoría de la enseñanza y desa- } \\
\text { rrollo del currículo }\end{array}$ & Madrid: Anaya \\
\hline 3 & 1983 & Blázquez, F. y Sáenz, Ó. & Didáctica General & Madrid: Anaya \\
\hline 4 & $\begin{array}{l}1987 \\
1995\end{array}$ & Zabalza, M. Á. & Diseño y desarrollo curricular & Madrid: Narcea \\
\hline 5 & 1988 & Gimeno, J. & $\begin{array}{l}\text { El currículo: una reflexión } \\
\text { sobre la práctica }\end{array}$ & Madrid: Morata \\
\hline 6 & 1989 & $\begin{array}{l}\text { Gimeno, J. y Pérez } \\
\text { Gómez, Á. }\end{array}$ & $\begin{array}{l}\text { La enseñanza. Su teoría y su } \\
\text { práctica }\end{array}$ & Madrid: Akal \\
\hline 7 & 1992 & $\begin{array}{l}\text { Gimeno, J. y Pérez } \\
\text { Gómez, Á. }\end{array}$ & $\begin{array}{l}\text { Comprender y transformar la } \\
\text { enseñanza }\end{array}$ & Madrid: Morata \\
\hline 8 & 1993 & Torre, S. de la & Didáctica y currículo & Madrid: Dykinson \\
\hline 9 & 1994 & Angulo, J. F. y Blanco, N. & Teoría y desarrollo del currículo & Málaga: Aljibe \\
\hline 10 & 1994 & Sáenz, Ó. & $\begin{array}{l}\text { Didáctica General. Un enfoque } \\
\text { curricular }\end{array}$ & Alcoy: Marfil \\
\hline 11 & 1997 & Díaz-Barriga, Á. & Didáctica y currículum & Barcelona: Paidós \\
\hline 12 & 1997 & Rodríguez-Rojo, M. & Hacia una Didáctica crítica & $\begin{array}{l}\text { Madrid: } \\
\text { La Muralla }\end{array}$ \\
\hline 13 & 1998 & Escribano, A. & $\begin{array}{l}\text { Aprender a enseñar. Funda- } \\
\text { mentos de Didáctica General }\end{array}$ & Cuenca: UCLM \\
\hline 14 & 1999 & Escudero, J. M. & $\begin{array}{l}\text { Diseño, desarrollo e innova- } \\
\text { ción del currículum }\end{array}$ & Madrid: Síntesis \\
\hline 15 & 1999 & Martín-Molero, F. & $\begin{array}{l}\text { La Didáctica en el tercer } \\
\text { milenio }\end{array}$ & Madrid: Síntesis \\
\hline 16 & 2000 & Marhuenda, F. & Didáctica General & $\begin{array}{l}\text { Madrid: } \\
\text { De la Torre }\end{array}$ \\
\hline 17 & 2004 & Rodríguez-Rojo, M. & $\begin{array}{l}\text { Didáctica General: qué y cómo } \\
\text { enseñar en la sociedad del } \\
\text { conocimiento }\end{array}$ & $\begin{array}{l}\text { Madrid: } \\
\text { Biblioteca Nueva }\end{array}$ \\
\hline 18 & 2004 & Sevillano, M. L. & La Didáctica del siglo XXI & $\begin{array}{l}\text { Madrid: } \\
\text { McGrawHill }\end{array}$ \\
\hline 19 & 2004 & Heredia-Manrique, A. & Curso de Didáctica General & $\begin{array}{l}\text { Zaragoza: Prensa } \\
\text { Universitaria }\end{array}$ \\
\hline 20 & 2005 & Tejada, J. & $\begin{array}{l}\text { Didáctica-Currículum: diseño, } \\
\text { desarrollo y evaluación curricular }\end{array}$ & $\begin{array}{l}\text { Barcelona: } \\
\text { Davinci }\end{array}$ \\
\hline 21 & 2008 & $\begin{array}{l}\text { de la Herrán, A. y } \\
\text { Paredes, J. }\end{array}$ & $\begin{array}{l}\text { Didáctica General. La práctica } \\
\text { de la enseñanza en educación } \\
\text { infantil, primaria y secundaria }\end{array}$ & $\begin{array}{l}\text { Madrid: } \\
\text { McGrawHill }\end{array}$ \\
\hline 22 & 2008 & Bolívar, A. & $\begin{array}{l}\text { Didáctica y currículum: de la } \\
\text { modernidad a la postmodernidad }\end{array}$ & Málaga: Aljibe \\
\hline
\end{tabular}


Cristina MORAL SANTAELLA y Agustín DE LA HERRÁN GASCÓN

\begin{tabular}{|c|c|c|c|c|}
\hline 23 & 2008 & Sánchez Huete, J. C. & Compendio de Didáctica General & Madrid: CCS \\
\hline 24 & 2009 & Medina, A. y Mata, F. S. & Didáctica General & $\begin{array}{l}\text { Madrid: } \\
\text { Printice-Hall }\end{array}$ \\
\hline 25 & 2010 & Moral, C. & $\begin{array}{l}\text { Didáctica. Teoría y práctica de } \\
\text { la enseñanza }\end{array}$ & Madrid: Pirámide \\
\hline 26 & 2010 & Bolívar, A. & $\begin{array}{l}\text { Competencias básicas y } \\
\text { currículo }\end{array}$ & Madrid: Síntesis \\
\hline 27 & 2011 & Cantón, I. y Pino, M. & Diseño y desarrollo del currículum & Madrid: Alianza \\
\hline 28 & 2011 & Navarro, R. & $\begin{array}{l}\text { Didáctica y currículum para el } \\
\text { desarrollo profesional docente }\end{array}$ & Madrid: Dykinson \\
\hline 29 & 2011 & Lorenzo Delgado, M. & $\begin{array}{l}\text { Didáctica para educación in- } \\
\text { fantil, primaria y secundaria }\end{array}$ & $\begin{array}{l}\text { Madrid: } \\
\text { Universitas }\end{array}$ \\
\hline 30 & 2014 & Gómez, I. y García, F. J. & Manual de Didáctica & Madrid: Pirámide \\
\hline 31 & 2015 & Domingo, J. y Pérez, M. & $\begin{array}{l}\text { Aprendiendo a enseñar. } M a- \\
\text { nual práctico en Didáctica }\end{array}$ & Madrid: Pirámide \\
\hline 32 & 2015 & $\begin{array}{l}\text { Medina, A. y Domínguez, } \\
\text { M. C. }\end{array}$ & $\begin{array}{l}\text { Didáctica. Formación ini- } \\
\text { cial para profesionales de la } \\
\text { educación }\end{array}$ & Madrid: UNED \\
\hline 33 & 2019 & Moral, C. & $\begin{array}{l}\text { Competencias para el diseño y } \\
\text { desarrollo de experiencias de } \\
\text { aprendizaje en la formación } \\
\text { del profesorado }\end{array}$ & Madrid: Síntesis \\
\hline 34 & 2019 & $\begin{array}{l}\text { Paredes, J., Esteban, R. } \\
\text { M. y Rodrigo, M. P. }\end{array}$ & $\begin{array}{l}\text { Didáctica inclusiva y transfor- } \\
\text { madora }\end{array}$ & Madrid: Síntesis \\
\hline 35 & 2020 & $\begin{array}{l}\text { Medina, A., Herrán, A. de } \\
\text { la y Domínguez, M. C. }\end{array}$ & $\begin{array}{l}\text { Hacia una Didáctica huma- } \\
\text { nista }\end{array}$ & $\begin{array}{l}\text { Madrid: UNED- } \\
\text { Red Iberoameri- } \\
\text { cana de Pedagogía } \\
\text { (REDIPE) }\end{array}$ \\
\hline
\end{tabular}

Fuente: Elaboración propia.

\section{Resultados y discusión}

Los resultados se encuentran recogidos en las Tablas 2y 3, que muestran el tipo de autoría y la media y porcentaje de aparición de las cate- gorías analizadas. En el Gráfico 1 se reflejan, de forma visual y sintética, los resultados obtenidos en relación con el número de capítulos por temáticas y su posicionamiento en los textos.

TABLA 2. Tipo de autoría.

\begin{tabular}{|c|c|c|}
\hline Autoría & Frecuencia & Porcentaje \\
\hline Individual & 18 & $51.4 \%$ \\
\hline Coordinada & 17 & $48.6 \%$ \\
\hline
\end{tabular}

Fuente: Elaboración propia.

En relación con la primera categoría considerada, y para responder a la primera pregunta de investigación, el análisis realizado muestra que 17 textos (un $48.6 \%$ ) están coordinados y 18 (un 51.4 \%) están elaborados de manera individual. Los coordinados incluyen mayor di- 
versidad de enfoques, vocabulario, conceptos y puntos de vista, pero, a la vez, pueden ser contradictorios y dificultar el aprendizaje conceptual. Algunos presentan una acumulación de capítulos sin hilo conductor, ni sentido aplicado. Los coordinados por Cantón y Pino (2011), Gómez y García (2014), y Domingo y Pérez
(2015) son de los pocos que incluyen ejemplos de diseños didácticos. En los trabajos de Gómez y García (2014) y Moral (2019), los capítulos teóricos guardan una relación estrecha con los ejemplos finales de diseños didácticos. En algunos, tanto coordinados como individuales, se han detectado errores técnicos.

TABLA 3. Media y porcentaje de los indicadores analizados.

\begin{tabular}{|l|c|c|}
\hline Categoría de análisis & Media & Porcentaje \\
\hline N.o de capítulos por libro & 12.8 & \\
\hline Epistemología & 3.2 & $25.7 \%$ \\
\hline Diseño & 1.4 & $11.3 \%$ \\
\hline Profesorado & 0.4 & $3.4 \%$ \\
\hline Alumnado & 0.1 & $0.9 \%$ \\
\hline Contenidos & 0.3 & $2.7 \%$ \\
\hline Objetivos & 0.4 & $3 \%$ \\
\hline Competencias & 0.2 & $1.4 \%$ \\
\hline Mezcla & 0.4 & $3.4 \%$ \\
\hline Métodos & 1.4 & $11.3 \%$ \\
\hline Técnicas & 0.8 & $6.4 \%$ \\
\hline Recursos didácticos & 0.8 & $6.4 \%$ \\
\hline Evaluación & 1.3 & $10.6 \%$ \\
\hline Innovación-desarrollo docente & 0.9 & $7.3 \%$ \\
\hline Clima social del aula & 0.2 & $1.4 \%$ \\
\hline Ejemplos/experiencias & 0.5 & $4.8 \%$ \\
\hline
\end{tabular}

Fuente: Elaboración propia.

Con relación a las temáticas, el mayor número de capítulos es el dedicado a la epistemología de la didáctica (25.7\%), con una media de $3 / 4$ capítulos por libro. Le siguen los capítulos sobre diseño curricular (11.3\%) con una media de 2 capítulos por libro. La evaluación es también un elemento de interés con un $10.6 \%$ de frecuencia de aparición, con una media de 1 a 2 capítulos por texto. Las competencias aparecen interrelacionando los elementos curriculares desde el manual de Herrán y Paredes (2008), desapareciendo el enfoque analítico de los componentes curriculares y las síntesis de componentes de la enseñanza-aprendizaje. Los capítulos sobre el profesor/a tienen una importancia poco destacada (3.4\%). Los dedicados a los alumnos son aún más escasos ( $0.9 \%)$. El compendio de Sánchez Huete (2008) se detiene en actitudes y motivación. Los dedicados a metodología didáctica alcanzan un $11.3 \%$. La «didáctica de la creatividad» es un tema innovador, asimilable a la metodología didáctica, que aparece en dos capítulos (De la Herrán 
y Paredes, 2008; Sánchez Huete, 2008). Las técnicas y estrategias de enseñanza comienzan a tener una aparición frecuente a partir del manual de Lorenzo Delgado (2011). Se agrupan, bien en torno a metodologías y principios didácticos (Lorenzo Delgado, 2011; Navarro, 2011; Gómez y García, 2014; Domingo y Pérez Ferra, 2015), o bien en torno a procesos de aprendizaje (Moral, 2010, 2019), con una frecuencia de aparición de $6.4 \%$. Esta puntuación está aumentada por el manual de Paredes, Esteban y Rodrigo (2019), que recoge 23 capítulos sobre competencias didácticas, para trabajar distintos aspectos de interés profesional propios del día a día de un docente -como emociones, motivación, exámenes, talleres, etc.-, con una orientación metodológica. De otro modo, el $86 \%$ de las obras analizadas no tienen ningún capítulo específico relativo a técnicas o competencias prácticas. Los recursos didácticos, así mismo asimilables a la metodología didáctica, man- tienen una importancia destacada (6.4\%). La relación entre profesor y alumnos y el clima del aula es otro elemento poco atendido, con solo un $1.4 \%$ de frecuencia de aparición. Los objetivos y los contenidos tienen una frecuencia de aparición similar (3\% y $2.7 \%$ ), parecida a los que mezclan los componentes curriculares en torno a las competencias (3.4\%). El desarrollo docente (profesional y personal) está ligado a la innovación educativa. En Sánchez Huete (2008) y en Herrán y Paredes (2008) se incluye el «desarrollo personal del docente» junto al profesional. La innovación es relevante en todos los textos, con un 7.3 $\%$ de frecuencia de aparición y un promedio de un capítulo por libro. Los ejemplos sobre diseños didácticos, en ocasiones presentados como estrategias de innovación, son relativamente escasos: en un $82 \%$ no aparece ningún ejemplo, pero en el de Domingo y Pérez (2015) se presentan 10 ejemplos/experiencias, que aumentan el promedio.

GRÁfICo 1. Número de capítulos (entre 10) y posición por temáticas.

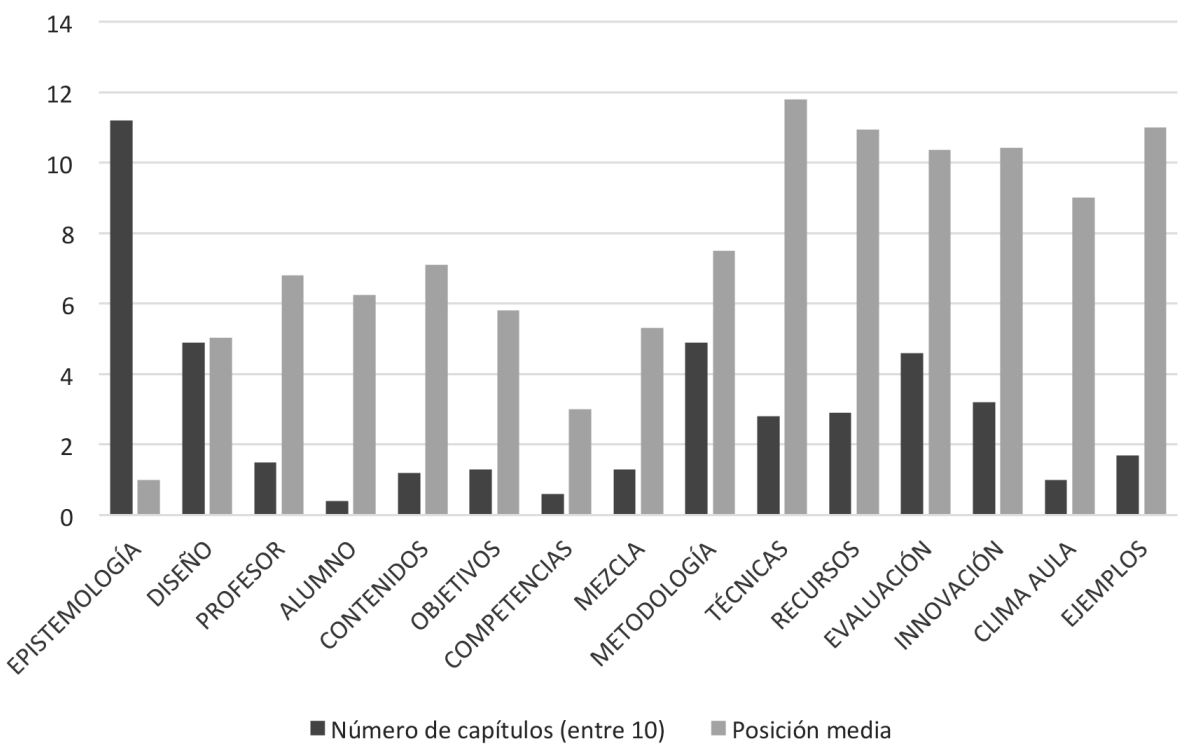

Fuente: Elaboración propia. 
En cuanto a la posición de los capítulos, un $88 \%$ comienza con una fundamentación de la Didáctica General. La segunda posición suele ocuparla el diseño curricular, el profesor o el alumno. Pocos comienzan con el profesor o con el alumno. En casi todos, los contenidos se tratan después de los objetivos. En los capítulos mezcla de elementos curriculares, donde los elementos curriculares se sistematizan en torno a competencias, los contenidos se colocan tras los objetivos y las competencias. El orden de los capítulos sobre elementos curriculares, así como en el interior de los capítulos mezcla, se sigue la tradición técnico-administrativa (Tyler, 1949): objetivos, contenidos, metodología-recursos y evaluación, como comenta Bolívar (2010).

A la vista de estos resultados, cabe preguntarse: ¿qué teoría de la enseñanza subyace y se transmite desde estos textos? La teoría (del griego the re [ver]) es necesaria, porque, sin teoría, no hay conocimiento, ni formación, ni práctica fundada. Pero la de estos textos, en general, no es suficiente para formar en competencias. En ellos, el componente teórico no competencial es muy alto. Los capítulos sobre epistemología, origen y fundamentos de la Didáctica y sobre teoría del currículo son los más numerosos en relación con el resto, y notablemente incomprendidos por los futuros profesores. $\mathrm{Pa}-$ reciera que la prioridad fuese la justificación de la Didáctica General y su conocimiento y el porqué de su existencia, excesivamente vinculada al currículo. Por otro lado, y no en pocos casos, los capítulos relacionados con las bases del diseño didáctico, en vez de facilitar su construcción, retornan a sus teorías, modelos de diseño curricular, complejidades e implicaciones, ocupando, en algunos casos, hasta 3 y 4 capítulos por libro, evitando la formación competencial aplicada.

A diferencia de otras disciplinas, la mayoría de los manuales no parece pretender la exhaustividad entre lo básico, de modo que casi todos dejan importantes parcelas sin abordar, viniendo a comunicar que el área no se ocupa de ellas. Otros, están sesgados desde su raíz por el ego del autor/a, de modo que solo trata lo que más le importa, sin el menor interés por descender a la práctica. Se deduce que muchas de estas obras, pese a su naturaleza, no son representativas de la disciplina, o bien solo lo son de alguna parcela u orientación de ella. La mayor parte son escasamente innovadores, en cuanto a capítulos epistemológicamente novedosos, lo que contradice las tesis que recogen en los capítulos sobre desarrollo docente e innovación educativa.

Nos preguntamos hoy, como hace 30 años, si es necesario que un maestro de infantil, primaria y secundaria tenga que conocer, con este grado de profundidad, la epistemología, los paradigmas, la bases, la dialéctica didáctica-currículo, la teoría del currículo, los modelos de enseñanza y de diseño... Hoy nos cuestionamos si tales contenidos, tratados de ese modo, son tan cruciales para su práctica competencial. Algunos parecen desarrollar un discurso no enfocado al nivel de enseñanza al que se destinan. Parecieran restos de proyectos docentes con una epistemología impropia, que en nada ayudará a mostrar la utilidad de la Didáctica General. Algunos incluyen contradicciones, incongruencias, errores, incluso didácticos. 
Los elementos curriculares más destacados y atendidos son la evaluación y la metodología didáctica. Los objetivos y los contenidos quedan, con diferencia, en un lugar posterior. La enseñanza por competencias ha hecho que los elementos curriculares aislados se agrupen en torno a ellas. Pareciera que el discurso didáctico de las competencias eclipsara el análisis de los otros elementos. Sin embargo, textos como los de Medina y Mata (2009), o Moral (2010, 2019), mantienen el análisis de aquellos elementos, sin el agrupamiento funcional. Es reseñable que, en los capítulos mezcla, estos se exponen uno a uno, lineal, secuencialmente y con poca interconexión mutua.

Sobre el orden de los capítulos, y dentro de la ya comentada secuencia tradicional (tyleriana), es relevante que los contenidos siempre suceden a los objetivos, y que la evaluación cierra la serie, incluso en la mayoría de los capítulos mezcla. Este dato refleja la vigencia, expresa o latente, de la tradición mantenida en España sobre los contenidos. Son pocos los investigadores del área que defienden el contenido, pues se entiende que mina la identidad de la Didáctica General, cuando esta disciplina está cuajada de contenidos propios. Tradicionalmente, ha habido una corriente que devaluaba los contenidos (Angulo y Blanco, 1994; Gimeno, 1981; Gimeno y Pérez Gómez, 1992). Rodríguez-Diéguez (1980) consideraba que el mayor mérito de las taxonomías de objetivos era «haber conseguido trasladar el centro de interés en la enseñanza de los contenidos e informaciones hacia las habilidades de distinto tipo» y, añadía, «asistimos, en el momen- to presente, a un renacer del «formalismo educativo", a una nueva preocupación por las capacidades formales más que por los contenidos» (p. 77).

\section{Gimeno (1981) decía que:}

La «educación centrada en el contenido» es el calificativo de la pedagogía llamada «tradicional». Para nosotros, la ventaja de separar objetivos de contenidos [...] está en que pone el énfasis en que el contenido es un medio para algo y no un fin en sí mismo [...]. Es decir, se relativiza el papel de los contenidos y se les pone al servicio de algo (p. 170).

Posteriormente, surgieron detractores del planteamiento de la «Pedagogía por Objetivos», en pro de las competencias. Bolívar (2010) abandona la programación por objetivos y considera que, aunque el enfoque por competencias no supone rechazar la importancia de los contenidos, ni conlleva un proceso de planificación didáctica, sí constituye una base para la elaboración especifica de los currículos: «Las competencias desempeñan un papel integrador, organizando los contenidos en función de lo que se espera que el alumno sea capaz de hacer. Como tales, reorganizan los elementos didácticos en función de lo que se quiere que adquiera» (Bolívar, 2010, p. 177).

Por el contrario, Zabalza (1995), defendió el lugar prioritario de los contenidos en el diseño didáctico:

La nueva escuela elemental, la española y la europea, se han de construir sobre una revalorización de los contenidos del 
aprendizaje (sobre una redimensionalización de la importancia del «saber») y de las técnicas de enseñanza-aprendizaje. Ya supongo que esta afirmación puede resultar polémica, y que el acuerdo en torno a esta cuestión no es general. Pero empieza a ser un principio común en toda Europa, y no precisamente (como algunos quieran para descalificarlo) entre los movimientos educativos conservadores, sino, más bien, en las posturas progresistas sobre educación. Habíamos llegado a tal laxitud con respecto a los contenidos que, para muchos, lo de menos era qué se estudiara, con tal que se hiciera de manera creativa, libre y agradable para los alumnos (p. 296).

Los anteriores comentarios ilustran, por contraste, la tendencia prioritaria de los textos de referencia de Didáctica General: aprender haciendo (Dewey, 1902). Las competencias, la acción, las distintas metodologías priman en los textos revisados. Difícilmente se aprecia en ellos la importancia de los contenidos para realizar los diseños didácticos (Zabalza, 1995). Solo el manual de Moral (2019) lo hace. Desde su perspectiva, centrarse en objetivos, competencias y actividades, en primer plano, es construir la casa desde el tejado. La acción, sin la formación y estructura conceptual en que cimentar el pensar, sentir y actuar, parece un sinsentido didáctico (Novak, 2010).

El profesorado de secundaria ha mostrado oposición y resistencia al enfoque de competencias, argumentando que minusvalora los contenidos (Bolívar, 2005). ¿Y los de primaria infantil? No reaccionan igual. ¿Es que en estos niveles no se construye una base de conocimiento sólida para pensar? ¿Qué se hace en estas eta- pas? ¿Actividades, jugar? Como destacan González-Sanmamed y Fuentes (2011), al hacer referencia a las creencias más arraigadas en la cultura docente, se llega a entender que: «enseñar es fácil, y prepararse para enseñar consiste en aprender a hacer las cosas (organizar juegos, realizar actividades)» (p. 55). Aunque, a la vez, resulte desconcertante: "con lo fácil que parecía enseñar y lo que cuesta que los alumnos aprendan» (González-Sanmamed, 2009. p. 71).

\section{Conclusiones}

Las quejas de los futuros docentes hacia enseñanzas teóricas excesivas, que no les preparan eficazmente para formarse en competencias docentes, parecen justificadas, desde el modelo de enseñanza competencial vigente. Se constatan al comprobar que, en las obras examinadas, la carga relativa de contenidos epistemológicos y teorías curriculares está descompensada, observándose una tendencia persistente a valorar los aspectos teóricos sobre los aplicados. Destaca la tendencia a considerar, en profundidad, los componentes curriculares de metodología didáctica y evaluación sobre otros con, al menos, la misma importancia, como la figura del profesor, a quien se dirige poca atención, o el alumno, olvidado casi por completo.

Los capítulos se ordenan, en general, según la tradición técnico-administrativa: epistemología y teoría curricular, objetivos, contenidos, metodología, y evaluación. Este orden refleja la prioridad real de los componentes curriculares, repercutiendo en la enseñanza de la programación didác- 
tica y, a su vez, en la adquisición del aprendizaje de competencias. En este artículo se ha planteado otro orden flexible y abierto a la innovación para el diseño didáctico que valora la memoria semántica y la construcción conceptual como bases del aprendizaje significativo, y el desarrollo de un pensamiento creativo y autónomo del alumnado (Novak, 2010; Sousa, 2017; Weinstein y Sumeracki, 2019). Este orden comienza por considerar primero al alumno, seguido del contenido, objetivos, evaluación, metodología y competencias. Poner en un lugar prioritario los contenidos no significa volver al modelo tradicional de contenidos criticado por Rodríguez-Diéguez (1980) 0 Gimeno (1981), ya que el contenido no es un fin en sí mismo, sin más. Por el contrario, la retención del contenido debe ser considerada un medio para propiciar un aprendizaje significativo, al servir de base para fundamentar la construcción de la estructura conceptual que sustenta la memoria semántica (Sousa, 2017).

Aunque se reconozca la limitada influencia de estos textos en la formación de un profesorado universitario en el ámbito de la Didáctica General, son obras de referencia epistemológica y de consulta básica y sensible para la formación de los docentes y la definición del área. La necesidad de encontrar y reconocer una orientación común es importante para la disciplina científica que fundamenta el conocimiento didáctico básico de los docentes (Shulman, 2005).

El conocimiento sobre el diseño didáctico es, a la vez, técnico y estratégico, y requiere combinar elementos armónica- mente vía innovación docente. Su coordinación e interdependencia ayudarían a comprender el proceso de diseño didáctico, tanto en el segundo nivel de concreción curricular como en las programaciones didácticas o de aula y en las unidades de programación didácticas y de otras propuestas metodológicas.

Por las razones anteriores, el diseño de un manual de Didáctica General debe cuidarse con esmero, en colaboración con las editoriales pedagógicas españolas, cuya tradición es de un alto nivel de realización. A tal fin, se proponen orientaciones discutibles para incrementar su educatividad y actualización conforme al modelo didáctico competencial, para propiciar experiencias fecundas de aprendizaje formativo en el alumnado:

- Conectar los manuales eficazmente con la relación de competencias profesionales, facilitando la construcción de un conocimiento didáctico fundamentado, práctico y profesional.

- Eliminar toda teoría rancia, superflua, ajena al interés y al ejercicio profesional de los futuros profesores.

- Vincular funcionalmente los capítulos teóricos y los que incluyen ejemplos de diseños didácticos.

- Pretender cierta exhaustividad de aspectos básicos, equilibrando temas de interés para el ego del autor con lo necesario para la formación práctica del alumno, y no dejar de incluir ámbitos claves del área, lo que podría 
comunicar desatención o negligencia disciplinar.

- Buscar una armonía epistemológica entre capítulos que evite conflictos conceptuales y de vocabulario, especialmente en las obras con varios autores.

- Ordenar los capítulos según una teoría de la enseñanza fundamentada, porque el orden de los factores sí afecta al producto.

- Evitar cualquier reflejo ideológico potencialmente manipulador 0 adoctrinador, en pro del máximo respeto didáctico a los alumnos, la formación del profesorado y el significado y sentido de la educación o de la formación.

- Atreverse a innovar, arriesgarse a incluir ámbitos innovadores, en la frontera del conocimiento didáctico, que apoyen el desarrollo epistemológico del área, en congruencia con las propias propuestas sobre innovación educativa.

- Validar el texto con expertos/as y un pilotaje adecuado, antes de publicarse, en aras de contribuir eficazmente a la formación en competencias, facilitando, de raíz, la construcción de un conocimiento científico, autocrítico, crítico, práctico y útil para la profesión docente.

- Proponer una enseñanza auténtica y coherente, habiendo puesto en práctica, previamente, lo que se propone al futuro docente.
Construir manuales de Didáctica General según estas condiciones podría aumentar su potencial profesionalizador y ayudar a reducir la distancia epistemológica entre la Psicología, la Neurociencia, la Didáctica General, las Didácticas Específicas y el Prácticum. Los fundamentos psicológicos y neurocientíficos del aprendizaje son requisitos para la formación didáctica de un futuro maestro. Una asignatura como Didáctica General no puede cursarse antes 0 simultáneamente a estas cuestiones, ni tampoco en el mismo cuatrimestre que las Didácticas Específicas. La formación de calidad en competencias didácticas requiere una vinculación especial de la Didáctica General y el Prácticum. Se propone reforzar este vínculo mediante instrumentos de observación, acción y evaluación válidos, fiables y de alta calidad, y la mayor coordinación 0 unificación de secciones de guías docentes de las asignaturas Didáctica General y Prácticum.

La situación descrita reclama una saludable autocrítica didáctica, de orientación autoformativa, radical, causal. La autocrítica, lejos de ser excepcional y fuente de conflictos para los profesores mediocres, debería ser un hábito científico y profesional, pues ennoblece a quien la hace. Si de formación se trata, el liderazgo de su práctica podría corresponder, en buena lógica, a los docentes-investigadores de Didáctica General. Pero no es fácil, por cuatro motivos relacionados con contenidos formativos no convencionales, asimilables a la Didáctica General: la dificultad para la autocrítica; el que la autocrítica solo tiene sentido si va seguida de rectificación 
(práctica), lo que requiere una madurez profesional especial; la tendencia del ser humano a pensar en términos duales, y la circunstancia de que se trata de afrontar un tradicionalismo didáctico persistente, cuyas raíces históricas terminan en nuestra mejorable formación.

\section{Referencias bibliográficas}

Anderson, L. W. y Krathwohl, D. R. (Eds.) (2001). A taxonomy for learning, teaching, and assessing [Una taxonomía para aprender, enseñar y evaluar]. Longman.

Bolívar, A. (2005). Conocimiento didáctico del contenido y didácticas específicas. Profesorado, 9 (2), 1-39.

Bolívar, A. (2008). Didáctica y currículum: de la modernidad a la postmodernidad. Aljibe.

Bolívar, A. y Pérez, P. (2019). Políticas educativas sobre el profesorado. En A. de la Herrán, J. M. Valle y J. L. Villena (Coords.), ¿Qué estamos haciendo mal en la educación? (pp. 25-35). Octaedro.

Consejo Escolar del Estado (2015). El profesorado del siglo XXI. Ministerio de Educación y Ciencia.

Council of the European Union (2018). Council recommendation of 22 May 2018 on key competences for lifelong learning [Recomendación del Consejo, de 22 de mayo de 2018, sobre las competencias clave para el aprendizaje permanente]. https://eur-lex.europa. eu/legal-content/EN/TXT/PDF/?uri= CELEX:32018H0604(01)\&rid=7

Darling-Hammond, L. y Oakes, J. (2019). Preparing teachers for a deeper learning [Preparando profesores para un aprendizaje más profundo]. HEP.

Dewey, J. (1902). The child and the curriculum [El niño y el currículum]. Chicago-Press.

Egido, I. y López, E. (2016). Condicionantes de la conexión entre teoría y práctica en el Prácticum de Magisterio. Estudios sobre Educación, 30, 217 237. https://doi.org/10.15581/004.30.217-237

European Commission (2004). Implementation of "Education and Training 2010» Work Programme. Key Competencesfor Lifelong
Learning. A European Reference Framework [Aplicación del programa de trabajo «Educación y Formación 2010». Competencias clave para el aprendizaje permanente. Un marco de referencia europeo]. https://step4-sfc.eu/IMG/ pdf/201509_a_integrer_dans_le_site_basicframe.pdf

Gairín Sallán, J., Díaz-Vicario A., del Arco Bravo, I. y Flores i Alarcia, O. (2019). Efecto e impacto de las prácticas curriculares de los Grados de Educación Infantil y Primaria. Educación XX1, 22 (2), 17-43. https://doi.org/10.5944/ educxx1.21311

González-Sanmamed, M. (2009). Una nueva oportunidad para la formación inicial del profesorado de Educación Secundaria. Revista de Educación, 350, 57-78.

González-Sanmamed, M. y Fuentes, E. (2011). El prácticum en el aprendizaje de la profesión docente. Revista de Educación, 354, 47-70.

Hall, R. (2020). Mixing methods in social research. Quantiative, qualitative and combine methods [Mezcla de métodos en la investigación social. Métodos cuantitativos, cualitativos y combinados]. Sage.

Hattie, J. y Clarke, S. (2019). Visible learning feedback [Retroalimentación del aprendizaje visible]. Routledge.

Heredia, A. (2015). Los manuales de Didáctica en español entre 1900-1970. revista española de pedagogía, 73 (260), 121-139. https://revistadepedagogia.org/wp-content/ uploads/2015/01/260-07.pdf

McTighe, J. y Willis, J. (2019). Understanding by design meets neuroscience [ $L$ a comprensión a través del diseño se une a la neurociencia]. ASCD.

Novak, J. (2010). Learning, creating and using knowledge [Aprender, crear y utilizar el conocimiento]. Routledge.

Ritchart, R. y Church, M. (2020). Making thinking visible [Visibilizar el pensamiento]. Jossey Bass.

Schwab, J. (1970). The practical 3: Translation into curriculum [La práctica 3: traducción al currículo]. School Review, 81 (4), 501-522.

Shulman, L. (2005). Conocimiento y enseñanza. Fundamentos para una nueva reforma. Profesorado, 9 (2), 1-28. 
Sousa, D. (2017). How the brain learn [Cómo aprende el cerebro]. Corwin.

Tyler, R. (1949). Basic principles of curriculum and instruction [Principios básicos del currículo y la instrucción]. Chicago-Press.

Walter, D. y Soltis, F. (2004). Curriculum and aims [Currículum y objetivos]. College-Press.

Weinstein, Y. y Sumeracki, M. (2019). Understanding how we learn [Entender cómo aprendemos]. Routledge.

\section{Biografía de los autores}

Cristina Moral Santaella es Profesora Titular de Universidad en la Universidad de Granada. Dedicada a la formación de profesores de infantil, primaria y secundaria, y a la investigación sobre distintos ámbitos de estudio para la mejora y el cambio educativo. Pertenece al Grupo de Investigación FORCE y a la Red RILME, entre otros. Sus líneas de investigación son la formación del profesorado, la didáctica y el currículo o el liderazgo educativo.

iD https://orcid.org/0000-0002-7302-165X

Agustín de la Herrán Gascón es Profesor Titular del Departamento de Pedagogía en la Universidad Autónoma de Madrid. Sus líneas de investigación son la formación del profesorado, la pedagogía de la creatividad, la historia de la innovación educativa, complejidad y conciencia en educación o el enfoque radical e inclusivo de la educación, entre otras. Pertenece al grupo de investigación PR-005 «Inclusión social y laboral de personas con discapacidad intelectual».

iD https://orcid.org/0000-0001-9156-6971 
